\title{
La rationalisation en France et en Belgique dans les années 1930
}

Rationalisation in France and Belgium in the 1930s

\section{Éric Geerkens et Aimée Moutet}

\section{(2) OpenEdition}

\section{Journals}

Édition électronique

URL : http://journals.openedition.org/travailemploi/2238

DOI : 10.4000/travailemploi.2238

ISSN : 1775-416X

Éditeur

DARES - Ministère du Travail

\section{Édition imprimée}

Date de publication : 30 décembre 2007

Pagination : 75-86

ISSN : 0224-4365

\section{Référence électronique}

Éric Geerkens et Aimée Moutet, "La rationalisation en France et en Belgique dans les années 1930 », Travail et Emploi [En ligne], 112 | octobre-décembre 2007, mis en ligne le 05 novembre 2010, consulté le 01 mai 2019. URL : http://journals.openedition.org/travailemploi/2238 ; DOI : 10.4000/

travailemploi.2238

\section{(c) La documentation française}




\title{
La rationalisation en France et en Belgique dans les années 1930
}

\author{
Éric Geerkens(*), Aimée Moutet(**)
}

\begin{abstract}
L'article compare l'introduction des méthodes nouvelles de l'organisation de travail («fordisme») et de la rationalisation de la production en France et en Belgique, dans un contexte de différences notables du tissu industriel entre les deux pays. Il approfondit les facteurs économiques et sociaux qui expliquent les différences constatables dans la mise en application des méthodes choisies. Des différences apparaissent au niveau du financement des entreprises, dans la structure industrielle, au niveau des droits de douane ou d'autres instruments de protection des marchés nationaux, enfin dans l'attitude de la part des partenaires sociaux (et principalement des syndicats) à l'encontre des méthodes nouvelles. Pour les auteurs, l'explication est à chercher dans l'environnement économique, qui semble plus lourd que les autres facteurs, et notamment que les positions des partenaires sociaux, dont l'influence n'a été qu'assez faible.
\end{abstract}

Dans l'étude du phénomène qui, sous le nom de rationalisation, a touché la plupart des industries occidentales dans la première moitié du $\mathrm{XX}^{\mathrm{e}}$ siècle, les rapports entre pays ont le plus souvent été examinés dans une perspective unidirectionnelle, mettant l'accent sur la diffusion, l'adoption adaptée des méthodes (américaines ou allemandes) d'organisation (Vaudagna, 1978; Jaun, 1986; Bloemen, 1988; Nolan, 1994). Comparer les choix d'organisation dans deux pays voisins, entretenant des relations étroites au cours du premier $\mathrm{XX}^{\mathrm{e}}$ siècle et également touchés par la crise des années 1930, conduit à s'interroger sur le poids des structures économiques et sociales dans les processus de changement organisationnel. Si la Belgique, petite économie ouverte qui exporte principalement des produits semi-finis issus des secteurs anciens d'industrialisation, n'est pas un terrain propice au développement d'une organisation qui peut être qualifiée de taylorienne-fordienne, alors que le France l'est davantage en raison de l'étendue de son marché intérieur et de l'importance de son industrie automobile, comment caractériser et expliquer le degré de pénétration de nouvelles méthodes d'organisation dans le tissu industriel des deux pays?

Partant de l'étude approfondie de pratiques d'organisation relevées dans l'ensemble des industries belge et française, il s'agit donc d'abord de comparer terme à terme les mesures de rationalisation prises, à l'échelle des entreprises, par ces industries touchées par la crise des années 1930, pour s'interroger ensuite sur les facteurs qui permet-

(*) Premier assistant à l'université de Liège, e.geerkens@ulg. ac.be.

(**) Professeur émérite à l'université de Paris-Nord, aimee. moutet@wanadoo.fr. tent de rendre compte des similitudes autant que des particularités nationales.

Pour conduire cette étude, des sources comparables ont été mobilisées de part et d'autre. Aux archives d'entreprises, dépouillées dans la mesure de leur conservation, meilleure en France qu'en Belgique, s'est d'abord ajoutée la presse technique et économique. Les rapports de stage des élèves des écoles de service social/surintendantes ont enrichi la vision de l'organisation interne des usines, alors que les archives et la presse syndicales renseignaient sur les réactions des travailleurs au changement organisationnel subi; des sources orales ont autant que possible été mobilisées.

\section{Les acquis organisationnels à la veille de la crise}

À la fin des années 1920, les acquis organisationnels dans les industries française et belge paraissent comparables, en dépit de cheminements différents mais partiellement croisés. Le paradigme de la production en série, impliquant l'usage des outils et pinces, le travail à tolérance, l'usage des calibres, s'est imposé, dès avant la Première Guerre mondiale, dans les grands ateliers de mécanique des deux pays (CoHen, 1994 et 2001; Barnier, 1998; GeERKENS, 2004a). Si la réception du taylorisme a été plus soutenue en France, où certains ingénieurs et industriels sont en relation directe avec Taylor, c'est l'expérience de la production de guerre qui différencie le plus significativement l'industrie française. En effet, alors que la demande des armées françaises favorise la diffusion du taylorisme dans les usines d'armement et la conversion des grands industriels 


\section{Encadré 1}

\section{La rationalisation: quelle définition? ( *)}

La polysémie du terme rationalisation, chez les contemporains comme dans les travaux historiques, appelle une clarification.

Entre les deux guerres, le sens du mot varie dans l'espace - il ne désigne pas les mêmes réalités en France et en Allemagne - comme dans le temps, la crise des années 1930 n'y étant pas étrangère.

Désignant d'abord des pratiques d'organisation introduites dans les ateliers avant, pendant et après la Première Guerre mondiale, son acception s'élargit pour rendre compte d'initiatives qui relèvent de plusieurs niveaux d'intervention - à l'instar de la normalisation/standardisation élaborée à l'échelle des branches et mise en œuvre dans les entreprises - ou qui, dans les entreprises, lient organisation du travail et gestion de la maind'œuvre, pensons aux surintendantes d'usine et aux formes de la politique sociale patronale dans les entreprises taylorisées.

Le concept de rationalisation prend également sens à l'échelle des économies nationales, dans une acception d'ailleurs différente selon qu'il s'agit des années 1920 ou de la période de crise. Dans les années d'expansion qui marquent la fin de la décennie d'après-guerre, elle désigne surtout des opérations financières de concentration alors que le désarroi provoqué par la crise fait résonner avec rationalisation une organisation moins désordonnée des activités économiques.

Pour mettre en évidence la cohérence d'un large ensemble de pratiques, observées d'abord à l'échelle des entreprises et à partir d'archives d'entreprises, nous avons choisi d'étudier la rationalisation comme l'ensemble des transformations de l'organisation matérielle de la production qui visent à accroître la productivité de divers secteurs de l'industrie belge et française entre les deux guerres.

\footnotetext{
(*) von HaAn H., 1938, «La terminologie et l'idéologie de l'organisation scientifique du travail», Revue internationale du travail, vol. XXXVII, nº 4, p. 449-470; KIRAT T., 1990, «Taylorisme et rationalisation du travail en France et en Allemagne dans l'entredeux-guerres", Revue d'économie politique, no 1 , p. 58-82 MOUTET A., 1998, «La rationalisation, une réponse aux problèmes de la seconde industrialisation (1880-1939)", Bulletin de la Société d'histoire moderne et contemporaine, $\mathrm{n}^{\circ}$ 1-2, p. 1-11.
}

de la construction mécanique aux principes fordiens de production de masse, l'occupation quasi complète $\mathrm{du}$ territoire belge paralyse assez largement l'industrie des métaux, dont les installations sont de surcroît détruites par l'occupant en retraite. Ces destructions ont certes permis un rééquipement favorable du point de vue technique, mais les structures industrielles sont restées dominées par les industries de base du XIX ${ }^{\mathrm{e}}$ siècle. Cela n'a bien sûr pas empêché la Belgique de développer certaines des industries nouvelles qui se répandaient alors en Europe. Au lendemain de la guerre, même si en France l'expérience de rationalisation menée au cours du conflit s'est poursuivie, des principes d'organisation largement hérités de l'avant-guerre ont subsisté, tout comme en Belgique: systèmes de salaire au rendement et réaffirmation de l'autorité des contremaîtres. Il faut attendre la période de conjoncture favorable consécutive à la stabilisation de 1926 en Belgique, de 1927-1928 en France, pour que les industries de ces pays s'engagent plus significativement dans l'adoption de nouvelles méthodes d'organisation. Ainsi, dans les deux pays, tout comme en Allemagne, les compagnies de chemins de fer taylorisent alors de manière systématique les travaux de réparation du matériel roulant, après avoir posé, dès avant la Première Guerre mondiale, les bases techniques d'une telle organisation (Peters, 1996; Moutet, 1997; Geerkens, 1998; CARon, 2003). De même, la forte croissance permet le financement d'une réorganisation en vue de l'introduction ou de l'extension du travail à la chaîne dans diverses industries (SChWEITZER, 1982; FrIDENSON, 1987).

En matière d'organisation, une collaboration s'est établie entre les industries belge et française tout au long des années 1920, s'appuyant sur des réseaux personnels ou institutionnalisés, dans le cadre notamment des participations prises dans les deux industries (BussiÈre, 1992; LANTHIER, 2004) et des échanges universitaires (BERTRAMS, 2006). L'ingénieur belge A. Galopin, qui a joué un rôle essentiel dans la fabrication en masse d'armes légères en France pendant la Première Guerre mondiale, avant d'orienter la rationalisation d'importants secteurs de l'industrie belge après le conflit, n'était-il pas un élève et disciple d'H. Le Chatelier, qui a introduit le taylorisme en France? Ce dernier tenait par ailleurs les laboratoires de la Fabrique nationale d'armes de guerre à Herstal (FN), où son influence s'exerce depuis l'avant-guerre à l'intervention de Galopin, pour le modèle du laboratoire industriel qu'il appelle de ses vœux (Le Chatelier, 2001). De même, l'introduction en Belgique de la psychotechnique comme pratique dans les entreprises et comme discipline dans l'enseignement supérieur doit beaucoup à P. Sollier, J. Amar et J.M. Lahy; notons que ce dernier a conduit une de ses premières «consultations industrielles» dans une entreprise parisienne, la MAP, contrôlée par la FN, avant d'organiser le service psychotechnique de l'usine herstallienne.

Enfin, l'organisation à Bruxelles, en octobre 1925, d'un Congrès international de l'organisation scientifique, a été l'aboutissement de la mise en place d'un comité d'organisation français sous le patronage d'H. Le Chatelier et de la société Michelin. Ce congrès a donné naissance aux deux comités d'Organisation Scientifique du Travail, français (CNOF) et belge (CNBOS). 


\section{Encadré 2}

\section{La psychotechnique (*)}

La psychotechnique est l'ensemble des méthodes psychologiques utilisées dans les transports et l'industrie pour sélectionner ou orienter le personnel en fonction des caractères du poste de travail et des aptitudes des postulants.

Apparues dès 1908, ces méthodes se sont développées dans l'entre-deux-guerres et surtout au cours des trente glorieuses selon trois orientations. La première ( $\mathrm{H}$. Laugier), dominée par les recherches physiologiques, visait à classer les hommes en différents types. La deuxième (J.M. Lahy) partait de l'analyse du travail en atelier; la définition de tests en laboratoire visant à mesurer les aptitudes n'intervenait qu'ensuite. La dernière, due à un ingénieur suisse, A. Carrard, reposait sur le dialogue et l'observation du sujet au cours de l'exécution des tests. II aboutissait à une estimation qualitative des aptitudes.

(*) LEPLAT J., «L'analyse psychologique du travail: quelques jalons historiques ", Le Travail humain, 1993, n² 2-3, p. 115-131; HUTEAU M., «L'École française de psychotechnique 1900-1940 », in CLOT Y. (sous la direction de), 1996, Les histoires de la psychologie du travail. Approche pluri-disciplinaire, Toulouse, Octares, p. 29-46; RABINBACH A., 2004, Le moteur humain. L'énergie, la fatigue et l'origine de la modernité, Paris, La Fabrique éditions, p. 445 et sv; MOUTET A., «La psychologie appliquée à l'industrie: un moyen de définition de l'aptitude ou de l'inaptitude? (de 1930 à 1960) », in Omnès C. et Bruno A.-S., 2004, Les mains inutiles. Inaptitude au travail et emploi en Europe, Paris, Belin, pp. 83-101.

\section{Les caractéristiques de la rationalisation de crise en France et en Belgique}

Après un bref rappel des caractéristiques structurelles des industries belge et française, il s'agit de comparer le degré de diffusion des pratiques d'organisation observées, pour relever ensuite les réactions ouvrières et syndicales qu'elles suscitent. La question qui se pose est bien de savoir si la crise des années 1930 a ou non maintenu la similitude des politiques de rationalisation dans les deux pays.

\section{Les pratiques d'organisation}

Pour comprendre certaines pratiques de rationalisation dans les industries française et belge touchées par la crise, un bref rappel de leurs caractéristiques structurelles s'impose.

L'industrie belge est très largement contrôlée par un nombre limité de banques mixtes et de groupes industriels (au premier rang desquels figure la Société Générale de Belgique). Ces groupes, engagés de longue date dans la vie industrielle du pays, ont d'abord pris le contrôle des secteurs qui ont fait la prospérité du XIX $\mathrm{XI}^{\mathrm{e}}$ siècle (principalement les charbonnages et la métallurgie). Au lendemain de 1'Armistice, ces groupes ont massivement réinvesti dans ces secteurs traditionnels, lucratifs en période de reconstruction. La dynamique industrielle repose alors principalement sur l'exportation de biens de production et de produits semi-finis, favorisée par une politique monétaire inflationniste, au détriment des secteurs produisant pour la consommation domestique. La stabilisation monétaire d'octobre 1926 modifie la dynamique sectorielle et favorise le secteur domestique, mais elle ne transforme toutefois pas radicalement la structure industrielle (CAssiers, 1989; CoOmans, 1989). Si l'extension du contrôle d'entreprises industrielles par des sociétés à portefeuille a amené une prédominance financière qui ne s'est pas accompagnée d'une centralisation du contrôle administratif et technique (VAN DER WeE, 1981), les plus importantes institutions financières et industrielles ont néanmoins ajouté au contrôle financier une surveillance technique, principalement dans le secteur charbonnier. Les inspections techniques de la Société Générale de Belgique et du groupe Coppée (surveillant ses propres exploitations et celles de la Banque de Bruxelles) ont tenu un rôle important dans la réorganisation des exploitations charbonnières. En raison de ce contrôle, la rationalisation a eu, dans ce secteur, un caractère plus systématique en Belgique qu'en France. Peu de temps auparavant, les banques mixtes avaient encouragé une concentration industrielle, stimulée par une législation qu'elles avaient contribué à faire adopter. Cette concentration, conçue en priorité pour le secteur charbonnier, a largement touché l'électricité, la métallurgie et la chimie. Les liens entre banque et industrie sont bien différents en France. Du fait de l'inaptitude des banques et du marché financier à satisfaire ses besoins et de l'attachement de chefs d'entreprises comme André Citroën à leur liberté de décision, c'est l'industrie qui finance l'industrie, par le moyen de l'autofinancement, favorisé dans certaines entreprises après la guerre par les profits qu'avait générés la fabrication d'armement, ou grâce à la constitution de groupes. Il en résulte tout à la fois pour ces entreprises une indépendance et des difficultés financières récurrentes (retard de la production à la chaîne chez Renault après guerre, faillite de Citroën en 1934) (LÉVy-LEBOYER, 1980). Par ailleurs, plusieurs des grandes entreprises favorisées par leur contribution à l'effort de guerre, dont en particulier les constructeurs automobiles, produisent des biens de consommation, qui peuvent s'écouler sur un marché intérieur plus important et mieux protégé qu'en Belgique, en même temps que la dépréciation du franc leur ouvre les marchés extérieurs, jusqu'en 1927 du moins. 


\section{Encadré 3}

\section{Structure industrielle, commerce extérieur et rationalisation}

L'inégal développement des travaux de comptabilité nationale rétrospective, en particulier le retard de la Belgique dans l'estimation des valeurs ajoutées par branche, conduit à privilégier la comparaison des structures industrielles en retenant comme indicateur l'emploi industriel par branche. En utilisant les données des recensements français et belge de 1936 et 1937, on dispose d'une image qui synthétise la période étudiée et permet d'examiner l'hypothèse du retard de développement de l'industrie belge lié, entre les deux guerres, à la défense des investissements dans les secteurs industriels traditionnels du XIXe siècle.

Tableau 1

Part de l'emploi des secteurs de base de l'industrialisation belge dans la population active industrielle totale de la Belgique et de la France, 1936-1937

\begin{tabular}{|l|c|c|}
\hline & $\begin{array}{c}\text { France } \\
(\mathbf{1 9 3 6})\end{array}$ & $\begin{array}{c}\text { Belgique } \\
(\mathbf{1 9 3 7 )}\end{array}$ \\
\hline Mines et minières & $4 \%$ & $11 \%$ \\
\hline Textiles & $11 \%$ & $15 \%$ \\
\hline Métaux, dont: & $20 \%$ & $17 \%$ \\
\hline - sidérurgie et non ferreux & $2 \%$ & $5 \%$ \\
\hline - transformation des métaux & $18 \%$ & $12 \%$ \\
\hline Total & $35 \%$ & $43 \%$ \\
\hline
\end{tabular}

Sources: ToutAIN J.-C., «La population de la France de 1700 à 1959», in Cahiers de l'ISEA, janvier 1963, AF, n 3, suppl. 133, tableaux 91 à 135; recensement économique et social du 27 février 1937, t. I, Bruxelles, Office central de statistique, sd, p. 92-106 et t. II, p. $182-189$.

En comparant la part relative de certaines branches de la «transformation des métaux " (construction d'appareils électriques, d'automobiles, d'avions, de machines agricoles et de navires) dans la population active industrielle totale des deux pays, on peut voir que ces terrains d'élection des méthodes tayloriennes d'organisation pèsent moins en Belgique $(2,4 \%)$ qu'en France $(4,5 \%)$.

Les structures industrielles des deux pays sont également marquées par leurs débouchés. Alors que la France dispose d'un important marché intérieur et exporte, à la veille de la Seconde Guerre mondiale, environ $15 \%$ de sa production de biens, I'Union économique belgo-luxembourgeoise (UEBL) exporte à ce moment $59 \%$ de cette production. L'étroitesse du marché intérieur belge a souvent justifié la faible spécialisation de ses fabrications. Les travaux, déjà anciens, de Svennilson et Maizels montrent la plus faible part prise par les produits dits progressifs, - produits dont la demande mondiale croît fortement - , et par les produits finis dans les exportations de l'UEBL, comparées à celles de la France.

Tableau 2

Ventilation par catégorie des exportations de l’Union économique belgo-luxembourgeoise et de la France, 1929 et 1938

\begin{tabular}{|l|c|c|}
\hline & Union économique belgo-luxembourgeoise & \multicolumn{1}{|c|}{ France } \\
\hline $\begin{array}{l}\text { Produits finis manufacturés } \\
\text { en proportion du total des exportations }\end{array}$ & $37 \%$ & $47 \%$ \\
\hline & \multicolumn{1}{|c|}{$\mathbf{1 9 3 8}$} & $51 \%$ \\
\hline $\begin{array}{l}\text { Produits régressifs (textiles } \\
\text { et produits manufacturés divers }\end{array}$ & $43 \%$ & $35 \%$ \\
\hline $\begin{array}{l}\text { Produits stables (métaux } \\
\text { et chimie) }\end{array}$ & $46 \%$ & $15 \%$ \\
\hline $\begin{array}{l}\text { Produits progressifs (machines } \\
\text { et équipement de transport) }\end{array}$ & $11 \%$ & \multirow{2}{*}{$1 \%$} \\
\hline
\end{tabular}

Sources: Maizels, A., Industrial Growth and World Trade, Cambridge UP, 1971, p. 64; Svennilson, I., Growth and Stagnation in the European Economy, Genève, United Nations Economic Commission for Europe, 1954, p. 295.

Comme l'emploi industriel, la ventilation des exportations des deux pays montre que l'industrie française compte une part plus importante d'activités qui, en théorie, appellent l'application des méthodes d'organisation permise par le travail en série. Mais il convient toutefois de rappeler que l'exploitation des houillères, activité industrielle de base, a connu, dans les deux pays, des changements organisationnels très significatifs. 
Dès le début de la crise, l'excédent durable de l'offre de travail sur sa demande a permis aux entreprises des deux pays d'opérer une sélection du personnel à la faveur de son licenciement. Les préoccupations psychotechniques, difficilement introduites dans les procédures de sélection durant les années 1920, cèdent rapidement le pas devant une sélection en fonction du rendement ou de la docilité, qui renforce l'autorité des chefs subalternes. Seules les entreprises de transport ont investi dans l'installation d'un laboratoire psychotechnique, afin principalement de réduire les charges liées à la survenance d'accidents. Face à la crise, en Belgique comme en France, les entreprises ont mené une politique de déflation des prix et des salaires, la seconde accompagnant la première. La crise a appelé dans les deux pays le même usage des études de temps et méthodes, renforcé en Belgique à la suite des grèves à caractère révolutionnaire de 1932 qui ont donné un coup d'arrêt aux réductions conventionnelles de salaires. Les chronométrages ont été utilisés de façon systématique pour accroître les rendements sans augmentation correspondante des gains ouvriers. Les menaces de licenciement, brandies très brutalement par la maîtrise, assuraient la soumission du personnel concerné. Dans ce contexte les méthodes tayloriennes ont été adoptées dans les secteurs ou les entreprises qui les avaient jusqu'alors négligées. Cette extension a favorisé et a été favorisée par l'activité d'une société de conseil en organisation installée en France en 1927 et active en Belgique à partir de 1930: la société Bedaux, filiale de la multinationale américaine du même nom (Moutet, 1987; KreIs, 1992; KIPPING 1999).

Bedaux offrait en effet une méthode de fixation des normes de production qui promettait aux industriels une réduction du prix du travail humain et une élévation des rendements. Ses ingénieurs non seulement mettaient en place son organisation mais formaient également l'encadrement de l'entreprise pour qu'il poursuive cette tâche. Aussi le système s'est-il diffusé dans les entreprises à la fois très laboristiques et peu expérimentées en matière d'organisation. Les sources laissent penser que si les entreprises utilisatrices ont été proportionnellement plus nombreuses en France, le système a peut-être été mieux exploité en Belgique, notamment son volet qualification du travail. Témoigne de cette avance dans l'application du système Bedaux l'orientation prise au lendemain de la Seconde Guerre mondiale par les travaux de la Commission technique générale, dont le système de cotation des postes est directement inspiré du système Bedaux, à l'intervention de plusieurs membres de cette Commission qui étaient d'anciens « ingénieurs Bedaux».

Loin de freiner l'adoption de moyens de production de masse, la crise a au contraire favorisé leur emploi, en France comme en Belgique, avec un succès toutefois variable. En France, les trois grands de l'automobile (Citroën, Renault, Peugeot) ont visé avec des investissements inégaux, à accroître la fluidité de leur processus de production, mais la majeure partie des petits constructeurs ont fait faillite, comme Delage, tandis que Hotchkiss s'orientait de façon préférentielle vers la fabrication des armements, en sorte que les trois grands ont fini par fournir $80 \%$ de la production française d'automobiles. Ces constructeurs ont, en dépit de la crise, ou pour l'affronter dans le cas de Citroën, créé les longues chaînes de montage en flux continu, en fonction desquelles ils se sont efforcés d'organiser les ateliers d'usinage (aux plans quantitatif et qualitatif) pour assurer la fluidité du procès de production entier (SChWEITZER, 1982; FrIDENSON, 1986). En Belgique, où le secteur automobile s'est concentré à la fin des années 1920 et où les deux principaux constructeurs (la Minerva Motors et la FN) se sont alors dotés de chaînes de montage, la fabrication automobile s'éteint avec la faillite de Minerva et avec le retrait de la FN, consécutif à la levée de la protection douanière de la branche.

L'application des méthodes de production de masse a été commandée par les caractères spécifiques de l'industrie dans chacun des deux pays: importance de l'industrie automobile et structure concentrée au profit des trois grands en France, faiblesse de cette branche du fait de l'étroitesse de son marché en Belgique, qui contrastait avec le poids des constructeurs de matériel roulant de chemins de fer et de la construction métallique dans l'économie de ce pays.

Avec la crise, la chaîne gagne également du terrain dans la fabrication de biens de consommation courante dans les deux pays, tout comme par exemple aux Pays-Bas (Moutet, 1983; Geerkens, 2004a; Vermis, 2001). En France, elle s'est toutefois limitée aux industries de la chaussure et de la petite construction électrique et cela sous une forme réduite aux transporteurs mécaniques. En Belgique, elle a touché les branches de la confection de vêtements d'hommes, de l'appareillage domestique ainsi que le conditionnement des produits alimentaires et pharmaceutiques. Cependant dans un secteur comme la confection en masse de vêtements pour hommes, la chaîne n'évince pas entièrement l'organisation ancienne qui, à la marge, apporte de la flexibilité au système productif. L'exploitation des charbonnages a suivi une évolution similaire dans les deux pays avec l'adoption d'un mode d'organisation assimilable au travail à la chaîne: concentration interne des travaux sur des longues tailles cycliques à forte production, qui appellent, dans une grande interdépendance technique, un renouvellement du remblayage, du soutènement et des transports (Moutet, 1986; GeERKens, 2002).

Dans les industries belge et française, les années de crise ont favorisé l'emploi de méthodes de planning qui coordonnaient les différentes phases de fabrication dans les ateliers et assuraient un contrôle élargi de la production, intégré dans la 
gestion d'ensemble. La crise a également assuré la diffusion d'une technique d'organisation américaine, portée à la connaissance des industriels européens en 1930: le contrôle budgétaire (Moutet, 1987; Berland, Boyns, 2002). Celui-ci donne aux directions centrales des entreprises les moyens d'une surveillance financière des fabrications. Il leur permet également de subordonner les choix de production aux possibilités de commercialisation, dans une période où la recherche de débouchés constitue le problème majeur des entreprises.

Sur le versant de l'encadrement social de la population ouvrière, les années de crise ont conduit les entreprises à abandonner des pans entiers de leur politique d'œuvres sociales, tenus pour inutilement coûteux. Toutefois, en Belgique, où le patronat confronté à une très forte syndicalisation a développé pendant les années 1920 un réseau d'œuvres qui sont des répliques, matériellement plus avantageuses, des services offerts par le mouvement ouvrier (mutualités, caisses de chômage, coopératives patronales), la crise a favorisé le développement de ces œuvres (GeERKens, 2002). Dans un secteur et une région déterminés - l'industrie lainière verviétoise-le patronat belge a fait appel à un dirigeant d'organisations patronales du textile français, D. Ley, pour qu'il acclimate à une industrie où les rapports professionnels étaient, de longue date, paritaires, un type de rapports sociaux particulièrement autoritaires, en vigueur dans le ressort du consortium de RoubaixTourcoing (GEERKENs, 2004b).

\section{Réactions ouvrières et syndicales}

Dans les deux pays, les mesures de rationalisation ont pesé sur les conditions d'activité et d'existence des ouvriers. Si la question du chômage technologique, imputable aux changements technicoorganisationnels et non à la seule baisse d'activité, a fait l'objet de controverses, il n'est guère contestable qu'à l'échelle de branches ou d'entreprises, les mesures de rationalisation ont poursuivi un objectif de réduction d'emploi. En s'en tenant aux données macro-économiques, certes réexaminées (SCHOlLIERs, 1995), les ouvriers belges subissent une véritable perte de pouvoir d'achat, quand en France, prix et salaires semblent baisser de concert (Prost, 2002). Par contre, en se plaçant à l'échelle des branches et des entreprises, on peut observer des baisses de salaires réels dans les deux pays, en particulier dans les secteurs où la rationalisation a été la plus soutenue, comme dans les charbonnages ou l'industrie des métaux; cette observation est d'autant plus nette que le chômage partiel est pris en compte. Cette dégradation des conditions de travail a rendu difficilement supportable son intensification, notamment lorsqu'elle résultait de l'application du système Bedaux, ou qu'elle accroissait la pénibilité d'activités qui l'étaient déjà par nature, comme les travaux souterrains des mines.
Alors que le degré d'organisation des ouvriers différait significativement, les réactions syndicales à la rationalisation ont été comparables dans les deux pays, tant par leur prudence que par leur insuccès.

Après la Première Guerre mondiale, la syndicalisation connaît en Belgique une exceptionnelle progression; celle-ci s'appuie principalement sur une logique de services, que les pouvoirs publics encouragent par leurs subsides (VANTHEMSCHE, 1994). Entre les deux guerres, le taux global de syndicalisation oscille entre 26 et $38,6 \%$ et s'établit en moyenne à 31,2\% (VANDAELE, 2003); dans le secteur des mines, des métaux et du textile, - secteurs exposés à la concurrence internationale où la syndicalisation est importante - les taux de syndicalisation atteignent respectivement les valeurs suivantes, en moyenne entre 1920 et 1937: 45\%, $66,8 \%$ et $73,8 \%$ (GEERKENS, 2004 ). Au lendemain de 1'Armistice, les nouvelles méthodes d'organisation sont alors admises, notamment parce que le syndicalisme pense avoir la force de contrôler leur application. Si les syndicats belges adhèrent à la rationalisation, ils disposent alors des moyens d'en obtenir une contrepartie pour les travailleurs. En France par contre, le taux de syndicalisation chute fortement après 1920 et demeure faible jusqu'en 1936; les fédérations du sous-sol, des métaux et du textile de la CGT pourtant réunifiée (près de 90\% des syndiqués en 1937) n'assurent respectivement en 1935 que les taux de syndicalisation suivants: $13,7 \%, 3,9 \%$ et $4,6 \%$, avant «la ruée syndicale de 1936» (РRоsт, 2006). La faiblesse des organisations ouvrières françaises ne leur donne guère les moyens de poser de telles exigences, bien qu'elles affichent la même adhésion de principe à la rationalisation.

Face à la gravité de la crise, les organisations syndicales des deux pays n'ont guère engagé leurs membres à combattre par la grève l'introduction de nouvelles méthodes d'organisation. D'une manière générale, on observe en Belgique une baisse de $38 \%$ entre la moyenne annuelle du nombre de grèves des années 1922 à 1929 et celle des années 1930 à 1935; en France cette baisse atteint 46\% (de Wasseige, 1953; Getz-Girey, 1965). Cette évolution globale ne doit toutefois pas conduire à ignorer l'importance de certains conflits circonscrits dans le temps et l'espace, comme les grèves belges de 1932 déjà citées. Il faut attendre 1935 et l'amorce d'une reprise économique pour que la conflictualité reprenne, à propos notamment de la rationalisation, comme l'illustrent les conflits dans la métallurgie de la Loire, dans les mines du Nord-Pas-de-Calais ou du bassin de Charleroi. Dans les deux pays, les organisations syndicales réformistes ont situé leur réponse face à la crise à un niveau bien supérieur à celui de l'atelier: Plan du Travail pour les socialistes belges puis pour la CGT; organisation des professions chez les démocrates-chrétiens belges. Les deux pays se démarquent par les voies de formation de l'unité ouvrière: en France, dans le contexte de 
constitution d'un front commun politique, la réunification des réformistes et des communistes s'opère à partir de la base, alors qu'en Belgique, ce sont les socialistes et les démocrates-chrétiens qui se rapprochent, très prudemment, à partir du sommet des appareils syndicaux.

En dépit de la prudence syndicale, certaines caractéristiques des nouvelles méthodes d'organisation du travail ont régulièrement été contestées par la base ouvrière, en particulier lorsqu'elles impliquaient une intensification de l'effort mal compensée financièrement, ou qu'elles paraissaient attentatoires à la dignité des travailleurs, en raison d'une surveillance trop étroite ou du soupçon d'effort insuffisant qu'elles véhiculaient. Toutefois, à l'exception du système Bedaux, dont l'introduction amène de nombreux arrêts de travail, l'emploi ou l'extension du chronométrage, du planning ou du travail à la chaîne, n'est pas à lui seul source de conflit en période de crise.

La comparaison entre les cas belge et français montre que la conjoncture a pesé plus lourd que les caractères structuraux des organisations ouvrières dans les réactions de ces dernières face à la crise. La puissance des syndicats belges ne leur a pas permis de résister à la rationalisation de crise plus efficacement que les faibles organisations françaises. S'y ajoutaient, comme dans les années 1920, le déterminant constitué par l'idéologie de syndicats ralliés, dans les deux pays, au principe de la rationalisation.

\section{Le renouvellement de la rationalisation dans le prolongement de juin 1936}

Les mesures de rationalisation étudiées ayant été introduites dans une conjoncture économique et sociale particulièrement défavorable aux travailleurs, se pose la question de leur devenir dans un contexte modifié par les grèves de 1936 et la reprise économique. Les syndicats ont-ils alors cherché à profiter de leur victoire pour mettre enfin en cause la rationalisation dans l'un ou l'autre pays, voire les deux? Les entreprises ont-elles limité leurs pratiques rationalisatrices?

\section{La place de la rationalisation dans les événements de mai-juin 1936}

En mai-juin 1936, les pratiques rationalisatrices paraissent diversement mises en cause par les organisations syndicales françaises et belges. En France, l'absence de dialogue social et surtout la pression exercée par les cadres subalternes pour accroître les rendements en période de crise ont été particulièrement mal ressentis. Le pouvoir ouvrier retrouvé grâce à l'unité syndicale, au gonflement des effec- tifs de la CGT - entre 1935 et 1937 , les effectifs de cette organisation dans le secteur privé sont multipliés par 11,7 (Prost, 2006) - et au succès des grèves de juin, ont semblé offrir aux travailleurs une possibilité de revanche qu'ils ont tenté d'exploiter, au moins jusqu'en 1938. Mais seuls les mineurs ont obtenu l'interdiction du chronométrage prolongé de l'ouvrier en taille, qui supprimait de facto le système Bedaux; par contre, l'espoir de contrôler la rationalisation patronale s'est révélé illusoire dans l'industrie manufacturière. En Belgique, où les organisations syndicales mettent autant en cause la pression que les caisses patronales exercent sur les consciences ouvrières que la rationalisation proprement dite, l'attitude prise à l'égard de cette dernière est modérée. Dans les deux pays, une réduction de la durée du travail est réclamée à titre notamment de compensation des effets négatifs de la rationalisation; de même, l'instauration ou le relèvement du salaire minimum visait notamment à protéger, dans une certaine mesure, les gains ouvriers contre une érosion excessive par les méthodes de chronométrage ou assimilées.

\section{Quel renouvellement des méthodes d'organisation après 1936 ?}

La nécessité de répondre au renversement de la conjoncture, ainsi que le renchérissement des coûts salariaux lié aux dispositions sociales prises pour mettre fin à la grève, ont rendu plus impératif encore le recours aux mesures de rationalisation. Si pour l'essentiel, celles-ci approfondissent la logique des mesures prises à la faveur de la crise, la seconde moitié des années 1930 a vu apparaître dans les deux pays une nouvelle rationalisation dont la Seconde Guerre mondiale a différé l'épanouissement.

D’un côté, les services nécessaires aux entreprises pour répondre à leurs besoins d'organisation - enseignement de l'OST et conseil en organisation - ont connu un nouveau développement. De l'autre, les grandes entreprises rationalisées ont perçu les limites de leur logique d'organisation et la nécessité, pour obtenir un nouvel accroissement de productivité et un abaissement de leurs coûts de production, de changements structuraux. Deux expériences peuvent être relevées : en France chez Renault, entre 1937 et 1939, en Belgique aux Ateliers de constructions électriques de Charleroi (ACEC), en 1939. Les transformations y ont porté sur l'organigramme de l'entreprise afin d'accroître l'efficacité de la gestion en démultipliant les responsabilités par secteurs de production: départements chez Renault, centres de frais aux ACEC, dont les cadres dirigeants devaient se comporter en «gérants d'une petite entreprise autonome». Dans ces deux entreprises, progresse également l'idée que c'est au stade de la conception du produit que doivent être pensées les conditions d'une production économique. Aux ACEC, cet objectif est traduit par l'idée que les études de matériel doivent être à la 


\section{Encadré 4}

\section{Le cadre des relations industrielles en Belgique et en France à la veille de la Seconde Guerre mondiale (*)}

Les systèmes de relations industrielles belges et français peuvent sembler formellement proches; en pratique, ils connaissent une évolution assez différente pendant l'entre-deux-guerres.

En Belgique comme en France, l'année 1919 marque un tournant dans les relations collectives du travail.

Avec certains précédents, des conventions collectives sont conclues en Belgique dès 1919, en dehors d'un encadrement légal spécifique - qui ne verra le jour qu'avec l'arrêté-loi du 9 juin 1945 - mais souvent dans le cadre de commissions paritaires de branche - les principales étant les mines, la sidérurgie, la mécanique et le textile (secteur lui-même subdivisé par région et par produit) - qui vont se réunir régulièrement durant la période étudiée. Certes, l'acteur patronal a plus d'une fois imposé l'agenda par une politique de la chaise vide, mais il n'a pas cessé de reconnaître ses interlocuteurs syndicaux de branche, alors même qu'il refusait le plus souvent cette reconnaissance à l'échelle de l'entreprise. Nées pour mettre en œuvre une réduction de la durée du travail, les commissions paritaires ont surtout traité des salaires. À un niveau inférieur à la branche, des comités officiels de conciliation avaient pour fonction, dès 1926, de prévenir les conflits en mettant en contact, à un niveau très local, des travailleurs plus ou moins organisés et des employeurs. Le champ de la négociation collective s'élargit significativement après 1936; alors qu'en 1935, on comptait quinze commissions paritaires nationales et dix commissions paritaires régionales, on ne dénombre pas moins de quatre-vingt-seize nouvelles commissions entre juillet 1936 et mai 1940. Si pour Gérard Lyon-Caen, la «Belgique est la terre du paritarisme», c'est précisément au cours de l'entre-deux-guerres qu'elle acquiert cette caractéristique.

En France, la loi du 25 mars 1919 donne, dans le prolongement de l'économie de guerre, un cadre légal aux conventions collectives; en 1919 et 1920, elles seront principalement conclues pour encadrer la réduction de la durée du travail. Mais en raison de la scission syndicale et de l'hostilité patronale, le paysage social français ne s'enrichit alors pas de commissions mixtes, où siègeraient régulièrement des représentants des organisations représentatives des employeurs et des travailleurs des principales branches industrielles; le nombre de conventions collectives conclues décroît fortement après 1920. Dans le prolongement des grèves de mai et juin 1936, la loi du 24 juin 1936 réactive le cadre conventionnel: près de 5500 conventions sont transmises au ministère du Travail au cours des deux premières années d'application de la loi. Mais un article (31vb) de cette loi limite d'emblée l'autonomie des interlocuteurs sociaux en prévoyant, en cas de blocage de la négociation, un arbitrage des conflits par un délégué des pouvoirs publics; cette autonomie est encore entamée par les lois des 31 décembre 1936 et 4 mars 1938 qui organisent l'arbitrage des conflits collectifs. La prorogation des conventions collectives arrivant à échéance par les lois des 18 juillet 1937 et 11 janvier 1938, le refus patronal de conclure de nouvelles conventions collectives après septembre 1937, les interventions autoritaires des pouvoirs publics pour élever la durée du travail dans les industries de guerre, enfin la répression de la grève du 30 novembre 1938, réduisent singulièrement la portée du paritarisme, au sens de la négociation collective fondée sur l'autonomie des interlocuteurs sociaux.

\footnotetext{
(*) WALINE P., 1947, Les conditions de travail (notes de cours destinées aux élèves de l'IEP-Paris), Paris, Centre de documentation universitaire; Rıoux, J.-P., 1977, «La conciliation et l'arbitrage obligatoire des conflits de travail », in RÉmOND, R. et BouRDIN, J., Édouard Daladier, chef de gouvernement: avril 1938-septembre 1939, Paris, FNSP, pp. 112-128; LUYTEN, D., 1995, Sociaal-economisch overleg in België sedert 1918, Bruxelles, VUB Press; Jamoulle, M. et al., 1997, Le temps de travail. Transformations du droit et des relations collectives de travail, Bruxelles, Crisp; DIDRY, C., 1998, «La nouvelle jeunesse des conventions collectives: la loi du 24 juin 1936 », in Le CRom, J.-P. (sous la direction de), Deux siècles de droit du travail. L'histoire par les lois, Paris, éditions de l'Atelier, pp. 129-141; DIDRY, C., 2002, Naissance de la convention collective. Débats juridiques et luttes sociales en France au début du XXe siècle, Paris, éditions de l'EHESS.
}

fois moins nombreuses mais plus poussées, afin de replacer dans les diverses fabrications un plus grand nombre de composants déjà mis au point; à défaut de maîtriser la longueur des séries, il s'agit d'accroître la répétition des composants communs. Chez Renault, mais plus encore chez Peugeot et Citroën, l'objectif est de réduire la diversité des travaux en employant des composants plus standardisés, ce qui impliquait que la définition des modèles se fasse en collaboration avec les ateliers de production.

Enfin la France a découvert dans l'industrie automobile le lien inéluctable entre production et consommation de masse et donc la nécessité de définir le modèle en fonction des utilisateurs populaires visés. Aussi est-ce à la suite d'une étude de marché que les usines Citroën sous la direction de la société Michelin ont défini les caractères d'une toute petite voiture (TPV), qui devait correspondre aux désirs d'une clientèle modeste de caractère largement agricole (FridENSON, 1988; LOUBET, 1997). Ainsi s'achevait l'évolution qui conduisait à l'intégration de la production dans la commercialisation, en même temps que pouvait s'ouvrir l'âge de la consommation de masse.

La crise sociale de 1936 a redonné son importance à la politique patronale de gestion de la main-d'œuvre, qui conserve cependant un caractère ambivalent. D'une part, on observe un regain d'intérêt pour des pratiques adoptées à la fin des années 1920 pour renforcer le lien social dans 
l'entreprise (journaux d'entreprises, conseils de sécurité, etc.). Un aspect de cette gestion de la main-d'œuvre a particulièrement retenu l'attention patronale dans les deux pays : le rôle des contremaîtres, à l'attitude desquels est imputée une part de la tension montée dans les ateliers jusqu'au déclenchement des grèves de 1936. Dans les deux pays, la réponse patronale a été comparable: il s'agit de former les contremaîtres au commandement, dans la perspective d'en faire des agents de première ligne dans une politique de collaboration de classe. Parallèlement, certaines directions assignent à une catégorie d'agents - hommes-secteur chez Michelin et Citroën, techniciens sociaux chez Peugeot, membres de syndicats maison en Belgique -, la mission d'être à l'écoute des griefs ouvriers pour prévenir les conflits (COHEN, 1988; FOMBONNE, 2001; Geerkens, 2004a). Mais d'autre part, le patronat ne cesse de contenir ou de contester les formes de représentation ouvrière indispensables à la négociation collective. En Belgique, le patronat, qui accepte de négocier en commission paritaire, tente néanmoins de perturber la négociation collective en favorisant la formation de syndicats maison, appelés à siéger dans les organes paritaires dès lors que leur représentativité serait reconnue. C'est dans l'industrie du zinc que cette stratégie a été poussée le plus loin, sans toutefois aboutir (LUYTEN, 1995; GEERKENs, 2002). En France, à l'occasion de conflits d'entreprise, les directions empêchent souvent la négociation en renouant avec la pratique ancienne du licenciement des meneurs ouvriers; à partir de septembre 1937, le patronat français renonce à la signature de conventions collectives négociées avec des représentants ouvriers.

\section{Les attitudes ouvrières et syndicales à l'égard de la rationalisation à la veille de la Seconde Guerre mondiale}

En Belgique, dans le prolongement de la première Conférence nationale du travail - sorte d'équivalent des accords de Matignon - les pouvoirs publics ont favorisé le règlement paritaire des différends entre acteurs organisés. Le retour d'une conjoncture plus favorable, du moins jusqu'à la fin de l'année 1937, et la progression de la négociation collective ont ramené les organisations syndicales dans un esprit comparable à celui de la veille de la crise, quand la rationalisation était admise dans l'espoir des avantages qu'elle pourrait apporter aux travailleurs. L'attitude prise à l'égard du système Bedaux illustre cette évolution; en effet, lorsque ce n'est pas l'introduction du système qui est négociée d'emblée, ce sont les conditions de son acceptation par les ouvriers au terme d'un conflit. Au-delà du contrôle ouvrier sur l'application des nouvelles méthodes d'organisation, on a pu observer à la veille de la guerre une volonté syndicale d'être associé à l'introduction de ces nouvelles méthodes; aux ateliers de construction mécanique de la société Cockerill, la collaboration entre les délégués du personnel et la direction prend notamment la forme de voyages d'étude en commun (vers des entreprises des PaysBas et du Danemark), qui préfigurent les missions de productivité de l'après-guerre.

En France, la conflictualité demeure importante après 1936 à propos de l'organisation du travail dans l'industrie des métaux et dans les charbonnages; si les dirigeants syndicaux se montrent disposés à discuter des mesures susceptibles de favoriser une plus grande productivité dans le cadre de la législation sociale nouvelle, ils ne sont pas entendus par le patronat. Les conflits salariaux, relancés en 1937 par l'inflation, débouchent non sur des accords paritaires mais sur l'intervention d'arbitres désignés par l'État. Dans l'automobile, les délégués ouvriers perdent rapidement le pouvoir de discuter les dispositions prises pour restaurer les rendements; dans les charbonnages, les délégués sont pris en tenaille entre le patronat, qui réclame - et obtient en partie - le rétablissement de l'organisation adoptée pendant la crise, et la base ouvrière, qui entend fixer elle-même la production qu'elle juge réalisable par tous sans effort inhumain (MOUTET, 1995).

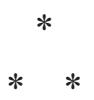

Dans les deux pays, la crise a assis d'une manière irréversible certaines conceptions de la productivité industrielle. La préparation taylorienne du travail ouvrier, la réduction des stocks en cours de fabrication par la fluidification du processus de production, par ailleurs objet d'un double contrôle, technique et financier, semblent acquises à titre de principe. Est-ce à dire que les industries de ces deux pays peuvent être tenues pour rationalisées à la veille de la Seconde Guerre mondiale? Posée d'une manière aussi globale, la question n'a guère de sens et, à défaut d'un corpus de sources représentatif, ne peut recevoir une réponse vraiment satisfaisante.

D'aucuns ont toutefois déjà apporté des réponses à la question, formulée en termes de structure industrielle et de spécialisation à l'exportation et à l'importation. Pour la Belgique, la réponse est plutôt négative. Pendant l'entre-deux-guerres, le poids des banques mixtes engagées dans les secteurs de base empêche une réallocation des investissements vers des secteurs livrant des produits plus finis, ce qui n'a toutefois pas interdit la fabrication d'un certain nombre de produits nouveaux; mais l'industrie belge largement tournée vers les marchés étrangers y exporte surtout des produits régressifs (dont la demande mondiale croît peu ou régresse) (HoGG, 
1986; CASSIERs, 1989). La situation française diffère par suite d'un engagement ancien dans les branches de la seconde industrialisation (construction automobile) relancé à la suite de la Première Guerre mondiale (industrie chimique organique et raffinage du pétrole) (CARON, Bouvier, 1980; SALY, 1986).

En se plaçant sur le terrain des pratiques d'organisation, une réponse nuancée peut être apportée.

De par leur proximité et leurs relations dans l'entre-deux-guerres, les deux pays présentent de grandes similitudes dans leur mode de rationalisation. En effet, depuis le début du siècle, les échanges culturels portés par les contacts humains ont favorisé le progrès de la rationalisation dans l'un et l'autre pays. Dans l'entre-deux-guerres en portent notamment témoignage l'évolution de la fabrication en construction mécanique et la diffusion de la psychotechnique. Dans le premier secteur, des échanges humains particulièrement fructueux ont été noués pendant la Première Guerre: des ingénieurs belges groupés autour d'A. Galopin ont, à l'occasion de la fabrication d'un important lot de fusils, montré aux industriels de la mécanique les conditions d'une production parfaitement interchangeable (Moutet, 2005); en retour, l'expérience faite en France de la production de masse d'armement a bénéficié à l'industrie belge après l'Armistice. Dans le second domaine, les rapports entre J.-M. Lahy et le docteur P. Sollier, praticien français enseignant en Belgique, a abouti dans les deux pays au progrès de la recherche comme de l'application de la psychotechnique, principalement dans les entreprises de transport; les deux hommes ont dirigé ensemble une revue, à l'existence certes éphémère, Science $d u$ travail. Psychotechnique et organisation, qui préfigure Le Travail humain lancé en 1933 par Lahy et Laugier (GEERKENS, 2004a).

Ainsi en dépit d'une structure industrielle qui globalement n'en constitue guère un terrain d'élec- tion, les méthodes tayloriennes s'implantent également dans l'industrie belge, en particulier dans les ateliers de construction et dans les ateliers de réparation et d'entretien du matériel ferroviaire. À l'instar de leurs homologues français, les industriels belges touchés par la crise retiennent principalement du taylorisme les aspects qui permettent de faire baisser les coûts salariaux sans impliquer de grands investissements dans l'appareil productif, comme la fixation des normes par le système Bedaux. La recherche d'une plus grande fluidité du processus de production, poursuivie dès les années 1920 dans divers secteurs de l'industrie belge comme française, rencontre un succès variable, qui tient pour partie à la taille et à l'ouverture des marchés nationaux. Si l'adoption des moyens de production de masse échoue en Belgique dans une fabrication emblématique, l'automobile, elle apporte, dans des secteurs mieux protégés, des résultats comparables à ceux enregistrés en France.

À la veille de la Seconde Guerre mondiale, s'esquissent dans les deux pays les modes d'organisation de la gestion et de la production qui se sont imposés après le conflit; en France l'industrie automobile reconnaît les règles d'une production de masse destinée à la consommation populaire. Par contre les rapports sociaux continuent à exclure toute collaboration entre organisations syndicales et patronat. Dans ces conditions, la France est restée le pays des conflits sociaux, où l'effort productif et le partage de son produit étaient fonction du rapport de force entre direction et personnel ouvrier dans l'entreprise. L'industrie belge semble offrir un profil inverse: restée plus traditionnelle par la nature de ses principales branches industrielles, le paritarisme, qui s'y était implanté au lendemain de la Première Guerre mondiale et s'était encore développé après 1936, était plus favorable à une grande mobilisation productive des travailleurs. 


\section{Bibliographie}

BARNIER F. (1998), «Aux origines du "taylorisme" à la française », Entreprises et Histoire, no 18, pp. 95-105.

Berland N., Boyns, T. (2002), "The development of budgetary control in France and Britain from the 1920s to the 1960s: a comparison", The European Accounting Review, $\mathrm{n}^{\circ} 11 / 2$, pp. 329-356.

Bertrams K. (2006), Universités \& Entreprises. Milieux académiques et industriels en Belgique, 1880-1970, Bruxelles, Le Cri édition.

Blemen E.S.A. (1988), Scientific Management in Nederland. 1900-1930, Amsterdam, NEHA.

Bussière E. (1992), La France, la Belgique et l'organisation économique de l'Europe 1918-1935, Paris, Imprimerie nationale.

CAssiers I. (1989), Croissance, crise et régulation en économie ouverte. La Belgique entre les deux guerres, Bruxelles, De Boeck.

CARon F. (2003), «À propos de la rationalisation du travail dans les ateliers des compagnies de chemin de fer en France», Revue d'histoire des chemins de fer, $\mathrm{n}^{\mathrm{0}} 28$ 29, pp. 190-206.

Caron F., Bouvier J. (1980), « Guerre, crise et guerre», in F. Braudel et E. Labrousse (éds), Histoire économique et sociale de la France. Années 1880-1950, Paris, PUF, t. IV (première édition Quadrige: 1993), vol. 2, pp. 648651.

Cohen Y. (1988), «Mais qui sont donc ces techniciens sociaux?», Vie sociale, no 2-3, pp. 41-55.

CoHen Y. (1994), «Inventivité organisationnelle et compétitivité. L'interchangeabilité des pièces face à la crise de la machine-outil en France autour de 1900 », Entreprises et Histoire, $\mathrm{n}^{\circ}$ 5, pp. 53-72.

CoHen Y. (2001), Organiser à l'aube du taylorisme. La pratique d'Ernest Mattern chez Peugeot, 1906-1919, Besançon, Presses universitaires franc-comtoises.

Coomans G. (1989), 1929. La crise en France, Belgique, Grande-Bretagne, Grenoble, Presses universitaires de Grenoble.

DE Wasseige Y.(1952), «La grève, phénomène économique et sociologique, étude inductive des conflits du travail en Belgique de 1920 à 1940», Bull. IRES, XVIII, no 7, pp. 663-724.

Fombonne J. (2001), Personnel et DRH. L'affirmation de la fonction personnel dans les entreprises (France, 1830 1990), Paris, Vuibert.

Fridenson P., (1986), “Automobile Workers in France and their Work 1914-1983", in S.L. KAPLAN et C.J. KeEPP (éds), Works in France, Ithaca N.Y., Cornell University Press, pp. 514-547.
FRIDENSON P. (1987), «Un tournant taylorien de la société française, 1904-1918», Annales ESC, pp. 1031-1060.

FridenSON P. (1988), «Genèse de l'innovation: la 2 CV Citroën », Revue française de gestion, septembre-octobre, pp. 35-44.

GeErkens E. (1998), «La rationalisation dans l'industrie belge: les réalisations de la Société nationale des chemins de fer belges (1926-1940)», Revue belge de philologie et d'histoire, 76-2, pp. 443-500.

GeErkens E. (2002), «Les mutualités professionnelles, un axe majeur de la politique sociale patronale pendant l'entre-deux-guerres en Belgique?», Revue belge de philologie et d'histoire, 80-4, pp. 1275-1349.

GeErkens E. (2002), «La rationalisation dans les charbonnages belges pendant l'entre-deux-guerres», in P. Wynants et H.-H. Herrman (éds), Huit siècles de charbonnage, Namur, CERUNA (colloques Meuse-Moselle, 2), pp. 339-365.

GeERKens E. (2004a), La rationalisation dans l'industrie belge de l'entre-deux-guerres, Bruxelles, Palais des académies (histoire quantitative et développement de la Belgique).

GeERKEns E. (2004b), «Une rationalisation autoritaire dans l'industrie textile: Verviers 1934-1936», in G.L. FonTANA et G. GAYот (éds), Wool: products and markets (13th-20th centuries), Padoue, Cleup, pp. 907-923.

Getz-Girey R. (1965), Le mouvement des grèves en France, 1919-1962, Paris, Sirey.

Hogg R.L. (1986), Structural Rigidities and Policy Inertia in Interwar Belgium, Bruxelles, Koninklijke Academie voor Wetenschappen, Letteren en Schone Kunsten van België.

JAUN R. (1986), Management und Arbeiterschaft: Verwissenschaftlichung, Amerikanisierung und Rationalisierung der Arbeitsverhältnisse in der Schweiz, 1873-1959, Zürich, Chronos.

KIPPING M., (1999), “American Management Consulting Companies in Western Europe, 1920 to 1990 : Products, reputation and relationschip", Business History Review, vol. 73, pp. 190-220.

KreIS S. (1992), "The Diffusion of the Scientific Management: The Bedaux Company in America and Britain, 1926-1945", in D. Nelson (éd.), Mental Revolution: Scientific Management since Taylor, Columbus, Ohio State University Press, pp. 156-174.

LANTHIER P. (2004), «Les dirigeants français du groupe Empain», in S. JaUmain et K. Bertrams (sous la direction), Patrons, gens d'affaires et banquiers. Hommage à Ginette Kurgan-van Hentenryk, Bruxelles, Le Livre Timperman, pp. 269-281. 
Le Chatelier H. (2001) (première édition 1925), Science et industrie: les débuts du taylorisme en France. Préface de M. LetTé, Paris, CTHS.

LÉVy-Leboyer M. (1980), "The large corporation in Modern France", in A.D. Chandler, et H. DAEms (éds), Managerial hierarchies; comparative perspectives in the crisis of modern industrial enterprise, Cambridge, Harvard U.P., pp. 117-160.

Loubet J.-L. (1997), «Citroën-Michelin et la crise : de la stratégie de survie à la stratégie de renouvellement (19351938)», in P. LANTHIER et H. WATELET (éds), L'entreprise privée en période de crise économique. Tactiques et stratégies, New York-Ottawa-Toronto, Legas, pp. 165179.

Luyten D. (1995), Sociaal-economisch overleg in België sedert 1918, Bruxelles, VUB Press.

LUYTEN D. (1996), Ideologisch debat en politieke strijd over het corporatisme tijdens het interbellum in Belgie, Bruxelles, Koninklijke Academie Voor Wetenschappen, Letteren en Schone Kunsten van België.

Moutet A. (1983), «Introduction de la production à la chaîne en France du début du $\mathrm{XX}^{\mathrm{e}}$ siècle à la grande crise de 1930», Histoire, économie et société, pp. 63-82.

Moutet A. (1984), «La Première Guerre mondiale et le taylorisme», in M. DE Montmollin et O. Pastre (éds), Le taylorisme, Paris, La Découverte, pp. 67-81.

Moutet A. (1986), «La rationalisation dans les mines du Nord à l'époque du Front populaire. Étude d'après les sources imprimées », Le Mouvement Social, n 135, pp. 63-99.

Moutet A. (1987), «Une rationalisation du travail dans l'industrie française des années trente», Annales ESC, septembre-octobre, $\mathrm{n}^{\circ}$ 5, pp. 1061-1078.

Moutet A. (1995), «Sous le gouvernement du Front Populaire: problèmes humains de la rationalisation et action ouvrière», in Y. CoHEN et R. BAudouï (éds), Les Chantiers de la Paix Sociale (1900-1940), Fontenay/ Saint-Cloud, ENS Éditions, pp. 287-310.

Moutet A. (1997), Les logiques de l'entreprise. La rationalisation dans l'industrie française de l'entredeux-guerres, Paris, EHESS.

Moutet A. (2005), «La rencontre des traditions militaires et civiles de rationalisation industrielle à l'occasion de la Première Guerre mondiale et ses conséquences »", in D. Pestre (sous la direction), Deux siècles d'histoire de l'armement en France. De Gribeauval à la force de frappe, Paris, CNRS Éditions, pp. 241-259.

Nolan M. (1994), Visions of modernity. American Business and the Modernization of Germany, New-YorkOxford, Oxford U.P.

Peters J.-H. (1996), «Rationalisierungsbestrebungen der Deutschen Reichsbahn-Gesellschaft zwischen 1924 und $1929 »$, Zeitschrift für Unternehmensgeschichte, 41, n 2 , pp. 187-200.

Prost A. (2002), «Les grèves de mai-juin 1936 revisitées », Le Mouvement social, no 200, pp. 33-54.

Prost A. (2006), Autour du Front populaire. Aspects du mouvement social au XX $X^{e}$ siècle, Paris, Le Seuil.

SAly P. (1987), «Poincaré keynésien?», in P. Fridenson et A. Strauss (sous la direction), Le capitalisme français aux XIX $X^{e}-X X^{e}$ siècles. Blocages et dynamisme d'une croissance, Paris, Fayard, 1987, pp. 33-46.

SCHOlliers P. (1995), “A century of real industrial wages in Belgium, 1840-1939”, in P. Scholliers et V. ZAmaGni (éds), Labour's reward: real wages and economic change in 19th and 20th century Europe, Alderschot, Edward Elgar, pp. 106-137.

Schweitzer S. (1982), Des engrenages à la chaîne: les usines Citroën 1915-1935, Lyon, PUL.

VAN DER WeE H. (1981), «La stratégie d'investissement des entreprises belges et son influence sur le développement économique de l'Europe», Revue internationale d'histoire de la banque, $\mathrm{n}^{\mathrm{0}}$ 22-23, pp. 1-22.

VANDAELE K. (2003), «De ontwikkeling van het sociaaleconomische overleg in het interbellum. De syndicale macht in de exportgerichte sectoren als een verklarende factor», Revue belge d'histoire contemporaine, $\mathrm{n}^{\mathrm{o}} 1-2$, pp. 129-176.

VANTHEMSChe G. (1994), Le chômage en Belgique de 1929 à 1940. Son histoire, son actualité, Bruxelles, Labor.

Vaudagna M. (1978), «L'américanisme et le management scientifique dans l'Italie des années 1920», Recherches, $\mathrm{n}^{\circ}$ spécial: le soldat du travail. Guerre, fascisme et taylorisme, pp. 389-432.

Vermis R. (2001), «Gedwongen tempo. De lopende band in Nederland tot de Tweede Wereldoorlog», NEHAJaarboek, pp. 227-257. 